\title{
A tentative fuzzy assessment of the quality of teaching and opportunities to learn mathematics in a classroom discussion
}

\author{
Itziar García-Honrado $^{1}$ Miquel Ferrer ${ }^{2}$ Angela Blanco-Fernandez ${ }^{3}$ \\ ${ }^{1}$ Universidad de Oviedo* \\ ${ }^{2}$ Universitat Autònoma de Barcelona \\ ${ }^{3}$ Universidad de Oviedo
}

\begin{abstract}
We study classroom discussions to obtain a fuzzy summary of the quality of teaching and opportunities to learn mathematics created in whole-class settings. We consider the ten rubrics of the Instructional Quality Assessment and make a fuzzy interpretation of this tool. We selected two problems of proportionality and collected data from forty-nine students. We make a statistical analysis of their written responses to the tool and consider their perceptions and feelings about the lesson. This analysis provided information about what rubrics are considered relevant to compute the fuzzy summary.
\end{abstract}

Keywords: Opportunities to learn mathematics, fuzzy summary, classroom discussions, Instructional Quality of Assessment

\section{Introduction}

This article studies a didactic problem, as it to evaluate an interactive classroom situation, in particular a classroom discussion, and the opportunities to learn mathematics that are created for the benefit of the students.

Our aim is to attempt to obtain a fuzzy set that summarizes the process of teaching and learning in a classroom discussion. Based on the relation of the quality of teaching and the generation of opportunities to learn, we base our study on a tool, the Instructional Quality Assessment (IQA), which combines ten rubrics that take into account the academic rigor and the accountable talk of a classroom discussion $[2,3,11]$.

Previous works (see, for instance, $[9,10])$ tried to partially fuzzify IQA rubrics because of the suitability of using fuzzy sets instead of crisp values to evaluate a classroom situation, which is full of imprecision and in which we consider that the perceptions of the teacher and students have an important role in the development of the lesson.

Based on the fuzzy descriptions of the participants' perceptions, we apply a fuzzy arithmetic

*Affiliated researcher to European Centre for Soft Computing. Email: garciaitziar@uniovi.es methodology [5] in order to obtain a weighted mean that aggregates all the aspects included in IQA.

This aggregation function allows us to obtain a fuzzy set that summarizes the quality of teaching in a whole-class setting. This kind of study was not previously done, since the original version of IQA does not consider any kind of aggregation for all the ten rubrics. However, it establishes a direct relation between great values in IQA and opportunities to learn. In particular, it suggests that as greater values a situation has in all the rubrics, a better situation is in terms of learning.

Finally, we propose to use this tool in some researches and in any classroom in order to favor teachers to have a critic spirit of their lessons.

\section{Previous concepts and goal}

Classroom discussions are a crucial resource for mathematics teaching, since they can facilitate the students' learning. Many teachers are used to organizing mathematics classrooms using these discussions, although their productivity depends on the number of learning opportunities that are created and how students can take advantage of these opportunities to learn. We assume that a classroom discussion is an instructional situation in which it is organized an oral reflection between students and teacher over a certain mathematical task. Interaction between participants may occur, although it will highly depend on the teachers' teaching activity when managing the lesson [7].

Episodes of a classroom discussion are defined through the articulation of two dimensions: the instrumental dimension, about the artefacts and how these are used in class [6], and the discursive dimension, about the interactional patterns that help to understand the generic development of the episodes and some of the particular characteristics shared among them [14]. More generally, we interpret episodes as systems of actions that have occurred in the course of the discussion. Our interest is on the effect of actions as some of them may foster procedural and/or conceptual mathematical learning [15].

The interpretation of classroom discussions in terms of sequences of episodes and actions has to do 
with our understanding of interaction as a crucial place for the development of mathematical learning. Various authors have studied the broader topic of opportunities to learn (see, for instance, $[4,11,12])$. In particular, we consider opportunities to learn mathematics as relationships between contents of mathematical knowledge, which are liable to be procedural and conceptual, together with actions that potentially contribute to facilitate the students' learning. These opportunities are identifiable through and from actions generated by diverse situations in the interaction processes of the mathematics classroom [7].

For the purpose of studying classroom discussions and opportunities to learn, in this article we present a study whose main aim seeks to investigate: How can we obtain a fuzzy summary of the quality of teaching and opportunities to learn mathematics in a classroom discussion?

\section{The classroom observation tool}

Our aim deals with the assessment of the quality of a classroom discussion and the study in depth of the opportunities to learn.

The methodology followed in these types of studies usually focuses on qualitative approaches, in which classroom discussions are firstly recorded and later transcribed and carefully analyzed to obtain interpretative conclusions. However, in many researches it is also useful to have quantitative tools to better confirm the results of the study. Particularly, for the case of classroom discussions, the wellknown tool is the IQA, which was introduced previously and whose set of rubrics is described below.

IQA allows teachers and researchers to assess a classroom discussion through the combination of ten rubrics, which refer to different didactic aspects that can be considered when analyzing a discussion. IQA rubrics are divided in two groups: Academic Rigor and Accountable Talk (see [11], page 659).

Academic Rigor is divided in five dimensions: Task Potential, that is, the cognitive demand of the task as it appears in the curricular materials; Task Implementation, that is, the cognitive demand of the task as it is implemented after students start to work on solving the task through the end of the lesson; Student Discussion Following the Task, which measures to what extent students show their work and explain their thinking about the important mathematical content; Questioning, that is, the type and amount of questions that the teacher provides students during the lesson; and Mathematical Residue, that is, the extent to which the classroom discussion builds new and/or important mathematical ideas.

Accountable Talk is also divided in five dimensions: Participation, that is, the percentage of students who participate in the classroom discussion; Teacher's Linking, that is, the teacher links between contributions within the discussion; Student's Linking, that is, the student links between contributions within the lesson; Teacher Asking, that is, the teacher press for conceptual explanations within the classroom discussion; and Student Providing, that is, the conceptual explanations provided by students within the discussion.

Each dimension is evaluated with a rubric that allocates values between 0 and 4 . For instance, for the case of Participation, the rubric is evaluated as follows:

0- If none of the students participated in the discussion.

1- Less than $25 \%$ of the students participated in the discussion.

2- Between $25 \%$ and $50 \%$ of the students participated in the discussion.

3- Between $50 \%$ and $75 \%$ of the students participated in the discussion.

4 - Over $75 \%$ of the students participated in the discussion.

And the rubric of the dimension Teacher Asking is defined as follows:

0- The classroom discussion was not related to mathematics or there was no discussion following the task.

1- There are no efforts to ask students to provide evidence for their contributions, and there are no efforts to ask students to explain their thinking.

2- Most of the press is for computational or procedural explanations of memorized knowledge, or there are one or more superficial, or trivial efforts to ask students to provide evidence for their contributions, or to explain their reasoning.

3- Once or twice during the lesson the teacher asks student to provide evidences for their contributions or explain their reasoning.

4- The teacher consistently (almost always) asks students to provide evidence for their contributions or to explain their reasoning.

The other rubrics of IQA and more information about this tool can be found in http://peabody.vanderbilt.edu/departments/tl/ teaching_and_learning_research/mist/ mist_instruments.php.

These rubrics, and for instance Participation and Teacher Asking, show two problems: the main one is that they try to evaluate imprecise things in a precise way. This leads into distinguishing between a discussion in which $74 \%$ of students participated and other in which the participation was $76 \%$ of students, or not distinguish between a discussion in which the $26 \%$ of students participated and other in which the $49 \%$ of students participated. The other problem appears because of using imprecision in the formulation of rubrics, as it happens when someone 
allocates 4 in the rubric of Teacher Asking. Then, what is the meaning of 'consistently'? Does it mean more than 'twice'?

The main cause of these problems is that concrete values in $\{0,1,2,3,4\}$ are allocated to imprecise situations, which are full of complex aspects that may change the valuation of each dimension. For instance, the way of asking students to explain their reasoning and the degree of depth the teacher expects to reach.

That is why we propose to use fuzzy sets to valuate these terms. As it is partially done in [9], we allow to allocate a fuzzy set to each dimension collected in IQA, based on the rubric proposed and the perception of all the aspects related to valuate it. For instance, in the case of Participation we can fix the number of students participating but also the way they participate, the frequency, the signs of non verbal participation,... All this information can be described into a fuzzy set, as it is shown in Figure 1. We propose to deal with trapezoidal fuzzy sets in order to simplify the way of describing perceptions by users and also to simplify the effectiveness of the program for computing the overall perception. On the one hand, the greatest base of the trapezium collects the values that are compatible with the user's belief, which will be the support of the graph (0-level). On the other hand, the smallest base (1-level) collects the values that are completely compatible with the user's perception.

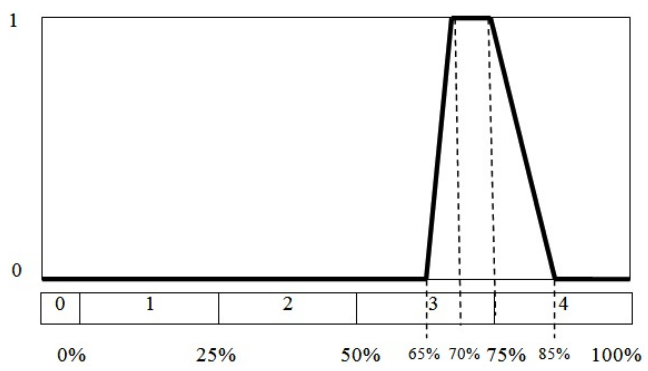

Figure 1: A fuzzy set valuating participation

Moreover, each fuzzy set could be translated into linguistic terms based on the linguistic variables: very low, low, medium, high and very high. This fact could be useful in two different ways: to complete the perception of each rubric based on the linguistic meaning, and to communicate the overall perception obtained with words.

A simple way to translate the overall perception into linguistic terms is comparing it with fuzzy sets that represent labels of the linguistic variables (see Figure 2) [20]. This comparison could be done by calculating indistinguishabilities [17] of the overall perception and fuzzy sets of the linguistic variable.

\section{Context and data}

We designed an instructional sequence of Geometry with similarity problems to be implemented over a

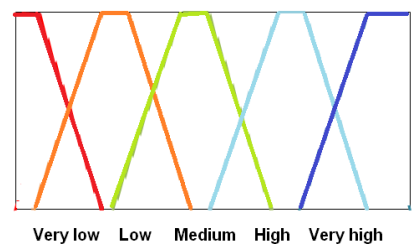

Figure 2: Linguistic variable

total of eight lessons. In this article we have selected two problems in the sequence to introduce the concept of proportionality, which is related to the notions of shape and similarity. Figure 3 shows the first problem, whose wording presents an open approach and whose resolution is tied to the activation of high cognitive tasks of proportional thinking [18]. There is more than one solution strategy and connections need to be made with the concepts of area and ratio.

Problem 1: Double figures!

Given the following letter from the alphabet, represent another letter that is twice the size. Below, briefly explain how you obtained it and compare it with the original.

Figure 3: Formulation of the first problem

Figure 4 shows the second problem, which was selected because it suggested students to work on a geometric transformation to turn the original triangle into another with twice the perimeter and move it on the plane. The two tasks were connected and the concept of homothecy with a positive ratio was introduced through a transformation that combined dilation and translation.

Problem 2: Similar triangles
How would you transform the polygon on the left
-1- to obtain the polygon on the right -2-? And
how would you transform the polygon 2 to obtain
the polygon 1? Explain both cases in detail.

Figure 4: Formulation of the second problem

Data was collected in December 2014 with fortynine students of a degree in education (pre-service teachers) in two classrooms, twenty-six students and twenty-three students, respectively. The work dynamics was collaborative and began with a paperand-pencil resolution of the two tasks in groups of four pre-service teachers during one hour. It continued with one-hour classroom discussion where all participants discussed the two mathematical activities. Finally, the pre-service teachers were asked to fill all the items of the ten IQA rubrics, each one described with a fuzzy set, to obtain their perception about the academic rigor and the accountable talk of the classroom discussion. In addition, they had to write down a fuzzy set that summarized their feelings over the whole lesson. After the discussion, participants' written materials were collected, both produced by working in small groups and individually after the lesson. 
After the classroom discussion, participants were asked to complete the rubrics of IQA. A questionnaire (see Figure 5) was given to students to complete eleven fuzzy sets: the first ten were linked to each rubric, respectively, and the last one corresponded to a summary of the overall perception of the quality of teaching in the classroom.

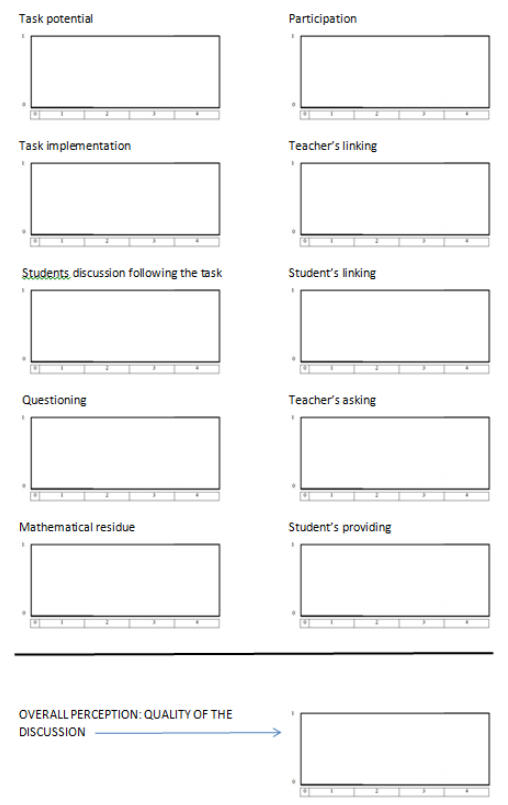

Figure 5: Questionnaire of perceptions

\section{Methodology}

The pre-service teachers' written responses to the IQA rubrics and their individual summaries with perceptions and feelings about the lesson were analyzed with a fuzzy statistical approach.

The ten rubrics were combined together to provide an overall perception of the quality of the classroom discussion. The overall perception of an individual is defined as a weighted combination of his/her rubrics, in order to take into account the different degree of explanation of each rubric to his/her general opinion about the classroom discussion.

Let us define the variable $X_{P}=$ overall perception of the classroom discussion and compute it as:

$$
X_{P}(\omega)=\sum_{j=1}^{10} \alpha_{j} X_{j}(\omega),
$$

for each individual $\omega$, where $X_{j=1}^{10}$ is the set of rubrics of the Instructional Quality Assessment and $\left\{\alpha_{j}\right\}_{j=1}^{10} \subset \mathbb{R}$ is a set of weights fulfilling $\sum_{j=1}^{10} \alpha_{j}=$ 1 .

Since $\left\{X_{j}\right\}_{j=1}^{10}$ are trapezoidal fuzzy sets, it is straightforward to show that $X_{P}$ is also a trapezoidal fuzzy set. As a weighted summary of the ten rubrics, it provides information about the general perception or opinion of each student on the classroom lesson.
To compute the weights $\left\{\alpha_{j}\right\}_{j=1}^{10}$, an intermediate statistical process has been developed. In the performed experiment, introduced in Section 4, an additional variable has been included in the IQA questionnaire. In addition to the ten rubrics, the students have been asked to give his/her answer to the following item: Overall perception: Quality of the discussion. This item has been also modelled as a trapezoidal fuzzy variable, whose possible values are trapezoidal fuzzy sets with support in $0 \%-100 \%$, analogously to the rubrics.

Let us define the variable $X_{G}=$ Overall perception: Quality of the discussion. Thus, the weights in (1) are computed in terms of the correlation coefficients of the ten rubrics with $X_{G}$ obtained from the sample data set, respectively (see Table 1 ).

In order to guarantee that the proposed process to compute the weights defining $X_{P}$ is statistically valid, some inferential studies are additionally developed. Namely, a hypothesis test is solved, to study if the expected values of the variables $X_{P}$ and $X_{G}$ are significantly different or not. If the latter situation holds, then it is concluded that the overall perception of the classroom discussion given by $X_{P}$ in (1), with the corresponding weights computed from the available sample data for $X_{G}$, effectively describes the general feeling of each individual about the session. Inferential results are shown in Section 6.1.

\section{Results}

Let $\left\{\left(X_{1}^{i}, X_{2}^{i}, \cdots, X_{10}^{i}\right), X_{G}^{i}\right\}_{i=1}^{n}$ the experimental dataset. Note that $n=49, X_{j}^{i}$ is the sample value of the rubric $j$ for the individual $i$, for each $j=1, \cdots, 10$, and $X_{G}^{i}$ is the sample value of $X_{G}$ for the same individual.

The correlation coefficients of $X_{j}$ and $X_{G}$, for all $j=1, \cdots, 10$, are computed by using the software package SAFD Statistical Analysis of Fuzzy Data, included in the statistical software R, with license GPL and downloadable from CRAN (see [19] for details). They are collected in Table 1. From those values, denoted by $\rho_{j}, j=1, \cdots, 10$, the weights $\left\{\alpha_{j}\right\}_{j=1}^{10}$ in (1) are computed as:

$$
\alpha_{j}=\frac{\rho_{j}}{\sum_{j=1}^{10} \rho_{j}} .
$$

The experimental values of $\left\{\alpha_{j}\right\}_{j=1}^{10}$ are also shown in Table 1.

\subsection{Testing the overall perception}

The sample values of the variable $X_{P},\left\{X_{P}^{i}\right\}_{i=1}^{n}$, are computed from its expression in (1) and the coefficients $\left\{\alpha_{j}\right\}_{j=1}^{10}$ provided in Table 1 .

The hypothesis testing procedure to test the equality of expectations of two fuzzy variables provided by the R-package SAFD [19] has been applied 


\begin{tabular}{|c|c|}
\hline$\rho_{j}$ & $\alpha_{j}$ \\
\hline .3467478 & .09468896 \\
.3846548 & .10504050 \\
.1925548 & .05258235 \\
.3242923 & .08855687 \\
.4843396 & .13226216 \\
.4325690 & .11812478 \\
.4030069 & .11005204 \\
.2859721 & .07809249 \\
.5407293 & .14766090 \\
.2671600 & .07293895 \\
\hline
\end{tabular}

Table 1: Experimental values of the weights

to solve the test:

$$
\left\{\begin{array}{l}
H_{0}: E\left(X_{P}\right)=E\left(X_{G}\right) \\
H_{1}: E\left(X_{P}\right) \neq E\left(X_{G}\right)
\end{array}\right.
$$

from the available dataset. The inferential algorithm approximates the $\mathrm{p}$-value of the test by $p=$ .585. Thus, the null hypothesis of equal expectations of the variables is not rejected at the usual significance levels $(1,5,10 \%)$. The sample means of $X_{P}$ and $X_{G}$ are shown graphically in Figure 6. It is immediate to see that they are trapezoidal fuzzy sets with similar behaviour. As a conclusion, the variable $X_{P}$ can be effectively used as a description of the overall perception about the classroom discussion, given the answers to the ten rubrics of the IQA.

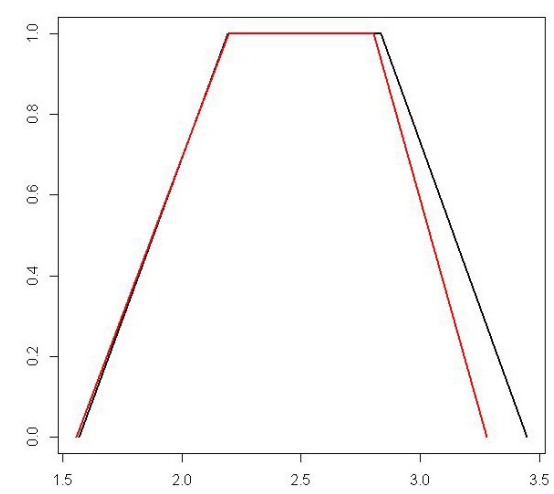

Figure 6: Sample means of $X_{P}$ and $X_{G}$

\section{Interpretation of weights}

Because of the coefficients obtained in the previous section, the overall perception is computed as a weighted arithmetic mean. In this section we try to interpret these weights analyzing what aspects of a classroom discussion are more relevant. Therefore, some hints for teachers can be shown in order to carry out high quality discussions.

The strongest correlated variable is one that depends on the teacher: Teacher's questioning. According to this result, more and deeper questions the teacher asks to their students, better quality of the discussion is obtained. In a similar way, Teacher's linking is another variable that has a high relation with the overall perception and contributes to involve students into the discussion, relating what the teacher wants to show to the student's previous knowledge.

Teachers in the process of asking and linking students' responses can help students to be more participative in classroom discussions. Students follow a leading role during classrooms because they can construct their own mathematical understandings [16]. This thesis is supported by the constructivist theory of education $[13,16]$.

All these facts are strongly related to the style of teaching [1]. Master lectures and non-participative classroom discussions seem not to be enough productive in the creation of opportunities to learn [7]. Therefore, we recommend teachers to organize open discussions, since they seem to obtain better results in the students' learning.

The Mathematical residue is, under our view, the effectiveness proof that the student makes the most of the discussion to understand and fixes the mathematical concept that the teacher wanted them to reach. This could be the reason of having a strong weight in the final weighted mean.

Finally, we want to point out that in this study we obtained the lowest values for the activities that depended on the students: Students discussion following the task, Student's providing and Student's linking. Therefore, this allows us to formulate an hypothesis: Is the teacher the member of a classroom discussion that has the strongest tools to lead the discussion into opportunities to learn?

\section{Application with GeoGebra}

GeoGebra (http://www.geogebra.org) is a software of dynamic geometry created for learning and teaching geometry in primary and secondary schools. Therefore, it is a well-known tool for primary and secondary teachers. That is why it could be a good media to show these fuzzy rubrics in schools to teachers in order to analyze the quality of a classroom discussion. The teacher could introduce their perceptions of a concrete lesson and obtain the summary through a fuzzy set that could be compared with the linguistic variables that were associated.

In [10] a tentative program using GeoGebra calculated the overall perception of a classroom discussion. However, it only took into account three of the ten dimensions of IQA and made the media of these three fuzzy sets. After the result obtained above, we can complete the overall perception by introducing all the dimensions of IQA, each one associated with its own weight and obtaining the overall perception.

In the screen of computers or tablets it appears the interface in Figure 7 and it allows teachers to introduce their perceptions of all dimensions of IQA. Teachers should just move the vertex of all the 
trapezoidal fuzzy sets in a friendly environment for them and, then, they could see a summary of the classroom.
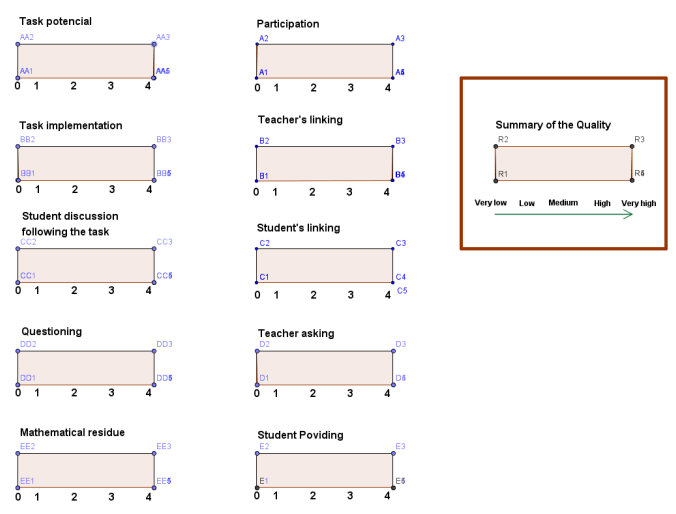

Figure 7: Fuzzy IQA in GeoGebra

\section{Application Example}

Some dimensions of IQA were studied previously in two particular classroom discussions with 14 or 15 -year-old students $[9,10]$, in which the previous similarity problems were discussed. In those studies it could be concluded that two discussions did not show the same opportunities to learn. Although they had similar values in many rubrics, the main differences were the way the problems were implemented by both teachers, the questions the teachers asked and participation. Our feeling is that one of these classroom discussions is better than the other, in terms of the amount of quality and opportunities to learn. After our analysis through the record of both classrooms and the design of fuzzy sets to each rubric in GeoGebra, we obtain the summary of both situations $^{1}$ (see Figures 8 and 9).

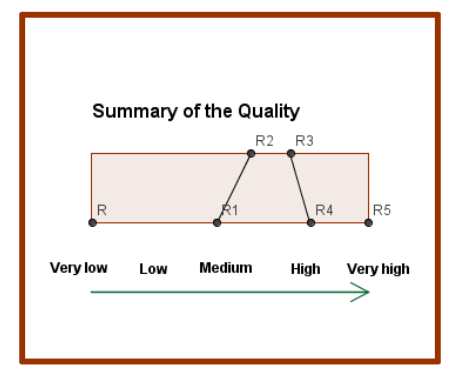

Figure 8: Sara's classroom discussion

Through the comparison of the fuzzy sets that summarize the quality of both classroom discussions represented in Figures 8 and 9, we can say that Sara's classroom discussion had higher quality than the one carried out by Luis, because the levels that Sara reached are higher than levels reached by Luis. We confirm our suspicious that before it

\footnotetext{
Luis.
}

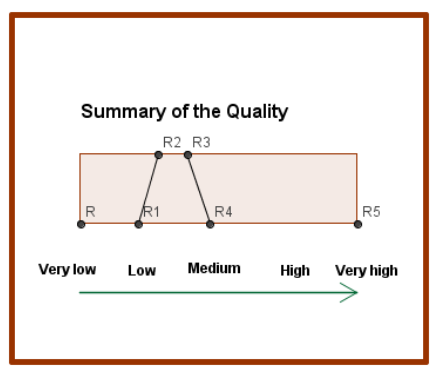

Figure 9: Luis' classroom discussion

was not so clear explained by the rubrics, and we can compare both situations by the comparison between two fuzzy sets.

\section{Conclusions}

In this paper we try to pass from a qualitative study, like the quality of a classroom discussion, to a quantitative study without losing the rigor of it. Furthermore, we want to obtain a mathematical summary of the process that involves many aspects related to discussions and opportunities to learn.

Firstly, we studied the well-known set of IQA rubrics, which allowed us to focus on the main aspects that we took into account to study the quality of the discussion. However, these rubrics, under our view, force situations to be evaluated with only four crisp values, losing a lot of information, and they do not allow teachers and researchers to obtain a summary of the quality of the lesson.

As in [9], the first problem was solved by using fuzzy logic. However, this paper focuses on obtaining a visual summary of the quality, taking into account all the aspects and dimensions of IQA. Each rubric contributes to compute the quality of the discussion through a weighted arithmetical mean, in which the weights are obtained by an experimental study based on forty-nine participants' perceptions, which were collected in this contribution.

The process of obtaining the weighted mean allows us to analyze what dimensions have a higher influence in the overall perception of the classroom. The actions leaded by the teacher to involve students in the discussion seemed to be very important. Therefore, some hints of the actions followed by the teacher are obtained and, in future works that could be related to the teacher's ways of acting [8].

What is more as the summary is a fuzzy set that has a visual interpretation, since the nearer the graph of the fuzzy set is to the right side of the horizontal axis, the better result is obtained, and even more linguistic variable helps to interpret the quality in linguistic terms.

Finally, this summary is not only useful in terms of research, it could be also popularized to make teachers conscious on the quality of the discussion they hold in their classrooms. 


\section{Acknowledgements}

This work has been partially supported by the Spanish projects EDU2011-23240 (M. Ferrer and I. García-Honrado), MTM2013-44212-P (A. BlancoFernandez), TIN2011-29827-C02-01 (I. GarcíaHonrado), and the grant FPI BES-2012-053575 (M. Ferrer).

\section{References}

[1] E. Aguilera, Los estilos de enseñanza, una necesidad para la atención de los estilos de aprendizaje en la educación universitaria. Review of Learning Styles, 10: 79-87, UNED, 2012.

[2] M. D. Boston and M. K. Wolf, Assessing academic rigor in mathematics instruction: The development of Instructional Quality Assessment Toolkit. Technical Report, National Center for Research on Evaluation, Standards, and Student Testing, Report No. 672, Los Angeles, CA, 2006.

[3] M. D. Boston, Assessing the quality of mathematics instruction, Elementary School Journal, 113(11): 76-104, The University of Chicago Press, 2012.

[4] D. J. Brewer and C. Stasz, Enhancing opportunity to learn measures in NCES data. In G. Hoachlander, J. E. Griffith and J. H. Ralph, editors, From data to information: new directions for the National Center for Education Statistics, pages 1-28, Washington DC: US Department of Education, 1996.

[5] S. de la Rosa de Saa, M.A. Gil, G. GonzalezRodriguez, M.T. Lopez, M.A. Lubiano, Fuzzy rating scale-based questionnaires and their statistical analysis. IEEE Transactions on Fuzzy Systems, 23(1): 111-126, IEEE, 2014.

[6] P. Drijvers, M. Doorman, P. Boon, H. Reed and K. Gravemeijer, The teacher and the tool: instrumental orchestrations in the technology-rich mathematics classroom, Educational Studies in Mathematics, 75: 213-234, Springer, 2010.

[7] M. Ferrer, J. M. Fortuny and L. Morera, Efectos de la actuación docente en la generación de oportunidades de aprendizaje matemático, Enseñanza de las Ciencias, 32(3): 385-405, UAB, 2014.

[8] M. Ferrer, J. M. Fortuny, N. Planas and K. Boukafri, Modos de actuación e interacción y generación de oportunidades de aprendizaje matemático. In M. T. González, M. Codes, D. Arnau and T. Ortega, editors, Investigación en Educación Matemática XVIII (SEIEM), pages 297-305, September 4-6, Salamanca (Spain), 2014.

[9] M. Ferrer and I. García-Honrado, Ejemplificación del uso de conjuntos borrosos en la valoración de la calidad de la enseñanza. In F. Bobillo, H. Bustince, F.J. Fernández and E.
Herrera-Viedma editors, Actas del XVII Congreso Español sobre Tecnologías y Lógica Fuzzy (ESTYLF 2014), pages 579-584, February 5-7, Zaragoza (Spain), 2014.

[10] I. García-Honrado and M. Ferrer, Valoración perceptiva de discusiones en gran grupo, $R e$ vista de Investigación e Innovación Educativa (in press), 2015.

[11] K. Jackson, A. Garrison, J. Wilson, L. Gibbons and E. Shahan, Exploring Relationships Between Setting Up Complex Tasks and Opportunities to Learn in Concluding Whole-Class Discussions in Middle-Grades Mathematics Instruction, Journal for Research in Mathematics Education, 44(4): 646-682, National Council of Teachers of Mathematics, 2013.

[12] X. Liu, Linking competence to opportunities to learn: Models of competence and data mining, Springer, New York, 2009.

[13] R. Mayer, Should there be a three-strikes rule against pure discovery learning? The case for guided methods of instruction. American Psychologist, 59: 14-19, 2004.

[14] L. Morera, N. Planas and J. M. Fortuny, Design and validation of a tool for the analysis of whole group discussions in the mathematics classroom. In B. Ubuz, C. Haser and M. A. Mariotti editors, Proceedings of the VIII Congress of European Research in Mathematics Education (ERME 2013), pages 1546-1555, February 6-10, Antalya (Turkey), 2013.

[15] M. A. Niss and T. Højgaard, editors Competences and mathematical learning: ideas and inspiration for the development of mathematics teaching and learning in Denmark, Roskilde Universitet, IMFUFA, 2011.

[16] A. S. Palincsar, Social constructivist perspectives on teaching and learning. Annual Review of Psychology, 45:345-375, 1998.

[17] J. Recasens, Indistinguishability Operators. Modelling Fuzzy Equalities \& Fuzzy Equivalence Relations. In Studies in Fuzzyness and Soft Computing, Vol. 206, Springer, 2011.

[18] M. K. Stein and M. S. Smith, Mathematical Tasks as a Framework for Reflection. Mathematics Teaching in the Middle School, 3: 268-75, 1998.

[19] W. Trutschnig and A. Lubiano, Package SAFD, CRAN R-project.org, 2013.

[20] L. A. Zadeh, The concept of a linguistic variable and its application to approximate reasoning - III. Information Sciences, 9(1): 43-80, Elsevier, 1975. 